\title{
Production Logistics Management of Industrial Enterprises Based on Wavelet Neural Network
}

\author{
Qian Liang \\ Shijiazhuang University of Applied Technology, Shijiazhuang 050000, China
}

Corresponding Author Email: liangqian@sjzpt.edu.cn

https://doi.org/10.18280/jesa.530418

Received: 9 March 2020

Accepted: 1 July 2020

\section{Keywords:}

wavelet neural network (WNN), industrial enterprise, production logistics, intelligent manufacturing

\begin{abstract}
With an efficient production logistics system, intelligent manufacturers can reduce the investment in production, improve the stability and self-repair ability of production logistics, and strike a perfect balance between production scheduling and production logistics. This paper probes deep into the production logistics management (PLM) of industrial enterprises, and proposes a PLM model for such enterprises based on wavelet neural network (WNN). Firstly, the PLM system architecture of industrial enterprises was established, and the scheduling and task allocation principles were proposed for the collaboration of various subjects in the system. Based on curved time window, a multiobjective path planning and optimization model was established, under influencing factors like the dynamics of station demand and the maximum driving range of handling equipment. Simulation results show that the proposed model is effective in optimizing the path for industrial production logistics. The research results provide theoretical supports to the real-time optimization of PLM and rationalization of production scheduling in industrial enterprises.
\end{abstract}

\section{INTRODUCTION}

In the era of intelligent manufacturing, the rapid response of the supply chain and the customization of products are the defining features of production. Multi-source information needs to be transmitted in the whole process of production, from material selection, production design, manufacturing, cargo transport, to sales. With the growing complexity of production processes, it is increasingly difficult to realize scheduling and collaborative control of the production logistics system (PLS) [1-3].

For intelligent manufacturers, an efficient PLS can effectively lower production investment, enabling them to provide quality consumer services more rapidly and timely. However, the existing PLSs encounter various problems, such as high transport cost, inaccurate scheduling, chaotic logistics routes, and frequent overstocks. To integrate production information and achieve intelligent, lean, and synergistic production, industrial enterprises must work hard to improve and optimize the operating efficiency of production logistics [4-7].

So far, fruitful research has been done on PLSs at home and abroad. The theories on logistics cost, logistics distribution, and Petri net have been applied to study the production logistics of enterprises [3, 5, 6, 8]. Zhang et al. [9] evaluated the impact of PLS on the production process, and highlighted the importance of reverse logistics to enterprise benefit, in terms of supply, distribution, and other aspects. Qu et al. [2] modeled he green PLS of intelligent manufacturers, using the Petri net based on production time series, and predicted the future trend of green production logistics in manufacturing. Stepanov [10] combined FlexSim simulation software with program analysis to present an effective solution that improves the operating efficiency of the production logistics in chemical plants. Following the just-in-time (JIT) theory, Wang and Ma [11] built a fuzzy evaluation model and an evaluation index system (EIS) for production logistics.

The operation mode of PLS constantly adapts to the changes in the external competitive environment. Therefore, more and more scholars are turning their attention to PLS optimization, especially the rational layout and implementation path [12-14]. Duan et al. [15] constructed a multi-objective optimization model for the implementation of PLS under complex conditions (e.g. machine constraint, time constraint, batch constraint, mixed flow constraint, and open constraint), and evaluated the stability of the model. Under the JIT-based flexible production condition, Santhosh et al. [16] proposed a mixed production and distribution scheduling method for manufacturers, which effectively reduces the production hours, distribution cost, and inventory cost. Sharma et al. [17] estimated the dynamic machine layout in various discrete jobshop areas with local feature algorithm, and verified the effectiveness of the algorithm in the comprehensive layout design of job-shops through simulation. Stepanov [18] created an evaluation system for the dynamic machine layout of jobshops, established optimization models for single-line and multi-line layouts, and proved the optimized layouts of production lines to be rational through case analysis. Relying on the multi-objective genetic algorithm (GA) based on difference calculation, Huang and Wang [19] designed the plane layout for the flow-shop of Ford Motor, and successfully elevated the efficiency of the PLS in the flow-shop.

The existing studies have improved the operating efficiency of production logistics to a certain extent. However, there are several defects with these studies: the uncertainty and dynamics of PLS are not fully considered, the global 
optimization of production logistics is difficult to achieve, and the time nodes of logistics links are not integrated into the management production logistics management (PLM) model. To improve the stability and self-repair ability of production logistics and perfectly balance production scheduling with production logistics, this paper explores the PLM model of industrial enterprises with the aid of the wavelet neural network (WNN). As an artificial intelligence (AI) technology, the WNN provides a desirable tool for information processing, due to its excellent self-learning ability and adaptability. The research results provide theoretical supports to the real-time optimization of PLM and rationalization of production scheduling in industrial enterprises.

The remainder of this paper is organized as follows: Section 2 built the architecture of the PLM system for industrial enterprises, and proposes the scheduling and task allocation principles of production logistics collaboration; Section 3 constructs a WNN for PLM of industrial enterprises; Section 4 establishes a multi-objective path planning and optimization model based on curved time window, in the light of various influencing factors; Section 5 proved the effectiveness of our model in industrial PLM and path optimization; Section 6 puts forward the conclusions.

\section{PLM ARCHITECTURE OF INDUSTRIAL ENTERPRISES}

For industrial enterprises, a complete PLM system (Figure 1) should cover a data source layer, a data acquisition layer, a data storage and processing layer, an application service layer, and a user management layer.

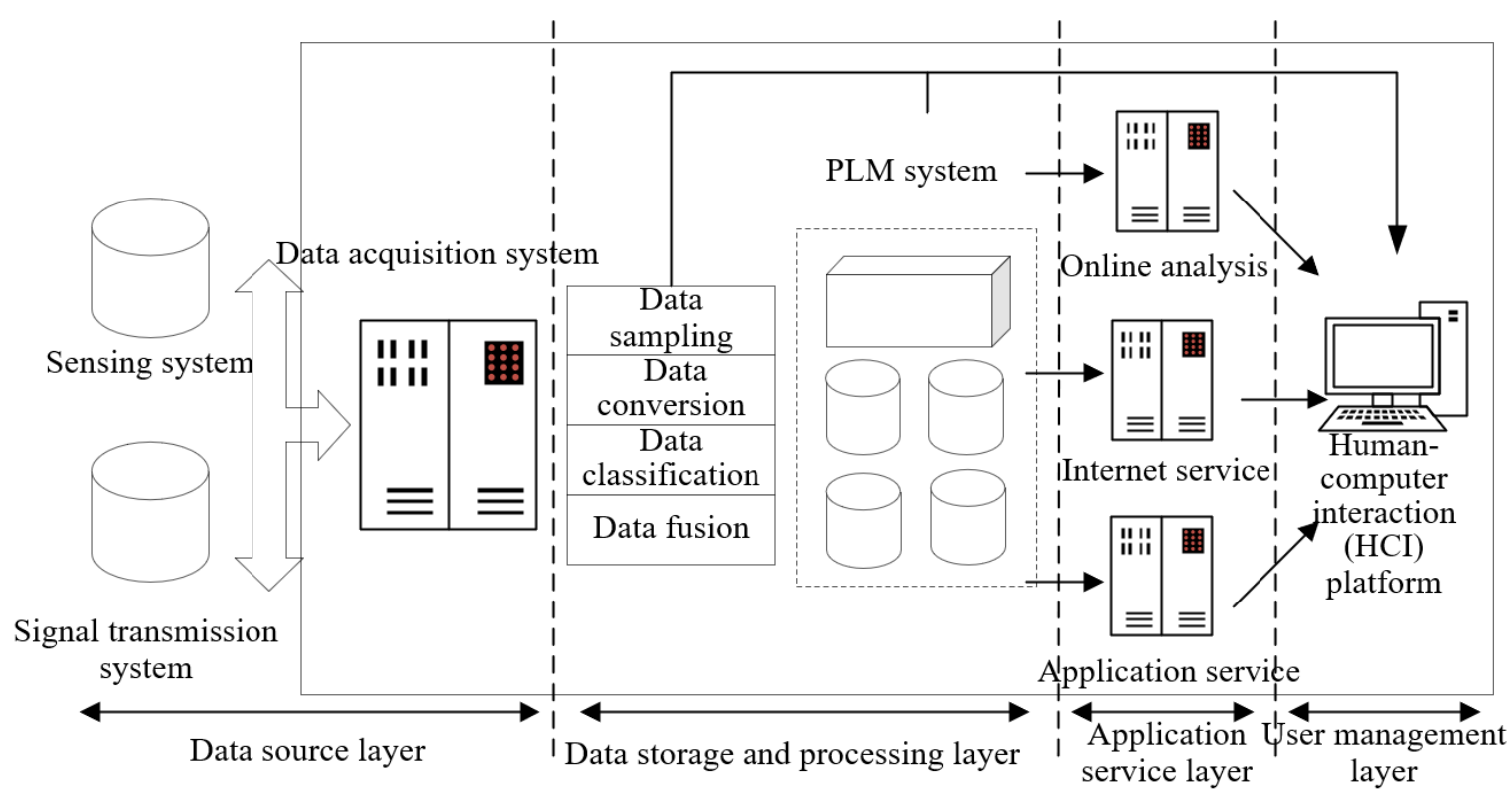

Figure 1. The PLM architecture of industrial enterprises

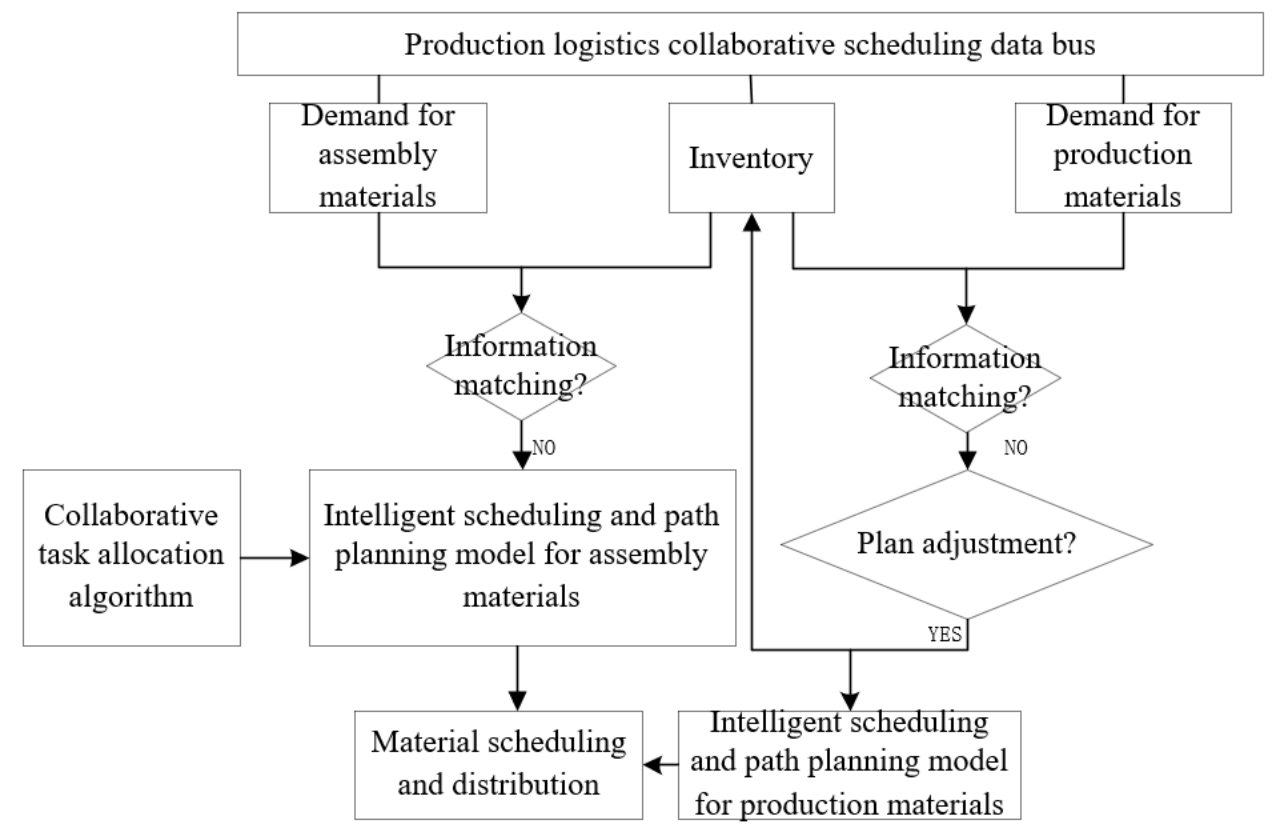

Figure 2. The scheduling and task allocation principles 
Based on advanced information technology (IT) advanced manufacturing technology, it is possible to realize all-round data interaction and attribute mapping between the PLM system in Figure 1 and the physical job-shops of modern industrial enterprises, thereby optimizing the process and efficiency of production control, and achieving the optimal effects on multi-machine collaboration, emergency handling, and logistic path planning. The PLM process can be divided into three steps:

(1) Based on radio-frequency identification (RFID), wireless sensor network (WSN), and various sensors, the realtime information (e.g. types of inventory materials, locations of materials and stations, the state of materials, and the path of handling equipment) is collected by the data acquisition module, processed through sampling, conversion, and fusion, and stored as the metadata of PLM system.

(2) If the physical job-shop has a dynamic demand, the PLM system will analyze the related data on the demand task, according to the information on the task and handling equipment, and make predictive planning of equipment scheduling and distribution path from the perspectives of feasibility, reliability, and safety;

(3) The predictive planning of the PLM system is simulated. Based on the simulation results, the managers make correct decisions through the human-computer interaction (HCI) platform.

Figure 2 provides the scheduling and task allocation principles of production logistics collaboration based on inventory materials, production equipment, assembly equipment, and handling equipment. If the inventory of assembly materials falls short of the demand of assembly task, the real-time inventory must be fed back to the intelligent scheduling and path planning model for assembly materials, and inputted to the predictive distribution planning model for assembly. If the inventory of production falls short of the demand of production task, the scheduling plan for production and assembly logistics should be adjusted by the collaborative task allocation algorithm, and the updated plan should be fed back to the predictive path planning models for production and assembly materials.

\section{PREDICTIVE PLANNING MODEL}

\subsection{Wavelet analysis}

Let $\mathrm{R}$ be the set of real numbers, and $S^{2}(R)$ be a vector space of a one-dimensional (1D) function whose squares are integrable. Then, the inner product of such a 1D function $g$ and wavelet function $h$ on the vector space $S^{2}(R)$ can be defined as:

$$
<g, h>=\int_{-\infty}^{\infty} g(x) \overline{h(x)} d x
$$

The norm on the vector space $S^{2}(R)$ can be defined as:

$$
\|g\|_{2}=\sqrt{\langle g, g\rangle}
$$

The fundamental wavelet refers to function $\varphi$, which belongs to $S^{2}(R)$ and satisfies the following admissibility condition:

$$
U_{\phi}=\int_{-\infty}^{\infty} \frac{|\widehat{\phi}(\tau)|}{|\tau|} d \tau<+\infty
$$

Under the constraint of the admissibility condition, function $\varphi$ is transformed into a window function with compact support and oscillation attenuation. Suppose $\mathrm{x}$ and $\mathrm{y}$ are nonzero real numbers. Then, the wavelet function $\left\{\varphi_{x, y}\right\}$ generated from function $\varphi$ can be expressed as:

$$
\phi_{x, y}(A)=\sqrt{|x|} \phi\left(\frac{A-y}{x}\right)
$$

where, $x$ and $y$ are continuous wavelets. In general, wavelet function $\varphi(x)$ is generated by the scaling function $\xi(x)$ :

$$
\left\{\begin{array}{l}
\xi(x)=\sqrt{2} \sum_{k \in I} q_{k} \xi(2 A-k) \\
\phi(x)=\sqrt{2} \sum_{k \in I} h_{k} \phi(2 A-k)
\end{array}\right.
$$

where, $I$ is the set of integers. The orthogonality constraint of (5), i.e. the relationship between the scaling function and the wavelet function, can be expressed as:

$$
h_{k}=(-1)^{k} q_{k}
$$

\subsection{WNN}

The biggest difference between WNN and traditional neural network $(\mathrm{NN})$ is the replacement of sigmoid function with nonlinear wavelet basis. The selected wavelet basis is translated to be superposed linearly with the scale factor, thereby expressing the signal. Based on the transform principle of discrete wavelet, the basis wavelet function $\varphi\left(a_{t-1}, \ldots a_{t-n}\right)$ satisfying the admissibility condition in Hilbert space can be determined by the following admissibility condition:

$$
\begin{gathered}
U_{\phi}= \\
\int_{S^{2}} \frac{\mid \widehat{\phi}\left(\tau_{a_{t-1},} \tau_{\left.a_{t-2}, \cdots, \tau_{a_{t-n}}\right) \mid}\right.}{\left|\tau_{a_{t-1}}\right|^{2}+\left|\tau_{a_{t-2}}\right|^{2}+\cdots+\mid \tau_{\left.a_{t-n}\right|^{2}}} d \tau_{a_{t-1}, \cdots, d \tau_{a-n}}< \\
+\infty
\end{gathered}
$$

The wavelet basis function can be obtained through the translation, rotation, and scaling of the fundamental wavelet:

$$
\begin{gathered}
\phi_{x, \alpha, \bar{y}}\left(a_{t-1}, \cdots, a_{t-n}\right)=x^{-1} \phi\left(x ^ { - 1 } \operatorname { r o t } _ { - \alpha } \left(a_{t-1}-\right.\right. \\
\left.\left.y_{x_{t-1}}, \cdots, a_{t-n}-y_{x_{t-n}}\right)\right)
\end{gathered}
$$

where, $x$ is the scale factor; $y$ is the translation vector; $\alpha$ is the rotation angle; $\operatorname{rot}_{-\alpha}=\left(a_{t-1}, \ldots, a_{t-n}\right)$ is the rotation vector:

$$
\begin{gathered}
\operatorname{rot}_{-\alpha}=\left(a_{t-1}, \cdots, a_{t-i}, \cdots, a_{t-j}, \cdots, a_{t-n}\right)= \\
a_{t-i} \cos \alpha-a_{t-j} \sin \alpha, 1 \leq i \leq j \leq n
\end{gathered}
$$

By properly adjusting $x, y$, and $\alpha, \varphi_{x, \alpha, \bar{y}}(*)$ can satisfy the frame properties of the following vector space:

$$
W_{1}\|g\|^{2} \leq \sum_{x, y}\left|<\phi_{x, \theta, \bar{y}}(*)>\right|^{2} \leq W_{2}\|g\|^{2}
$$

where, $W_{1}$ and $W_{2}$ are frame boundaries satisfying $0 \leq W_{1} \leq W_{2} \leq \infty$. Figure 3 presents the $\mathrm{WNN}$ designed for predictive planning of industrial production, assembly, and distribution.

The set of wavelet functions $\left\{\varphi_{x, \alpha, y}(*)\right\}$ is the activation number of the only hidden layer in the WNN. Rearranging this set into $\varphi_{1}(*), \varphi_{2}(*), \ldots, \varphi_{\mathrm{n}}(*)$, the WNN $g(*)$ can be approximated as: 


$$
\begin{gathered}
\hat{g}(*)=\sum_{i=1}^{N} \omega_{i} \phi_{i}(*)= \\
\sum_{i=1}^{N} \omega_{i} \phi_{x, \alpha, \bar{y}}\left(a_{t-1}, a_{t-2}, \cdots, a_{t-n}\right)
\end{gathered}
$$

where, $\omega_{i}$ is the connection weight between hidden layer nodes and output layer nodes; $\varphi_{x, \alpha, \bar{y}}(*)$ is the output of hidden layer nodes.

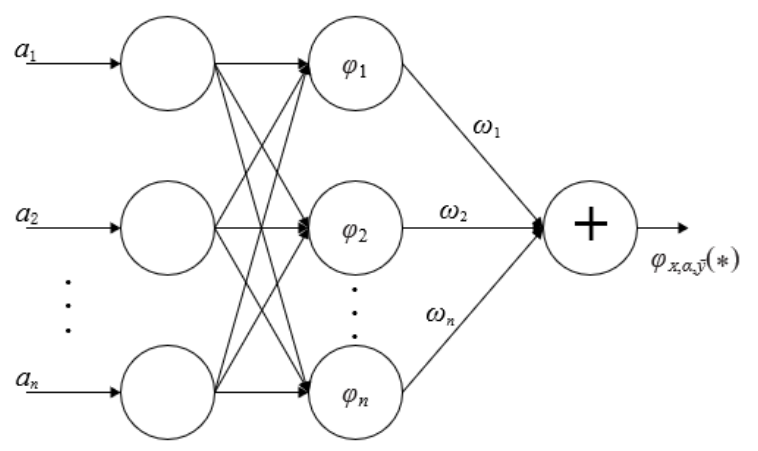

Figure 3. The structure of our WNN

\section{WNN-BASED PREDICTIVE PLANNING OF PRODUCTION LOGISTICS}

\subsection{Time window function}

To obtain the objective function of WNN-based optimization, this paper defines the effective time period during which the handling equipment is allowed to serve the production or assembly equipment. Normally, there are two kinds of time windows, depending on the strictness of the time constraint: the hard time window, i.e. the scheduling or distribution service must be completed in the required period, and the soft time window, i.e. the service can be completed in the best period.

In actual production, however, if the arrival time of the handling equipment deviates slightly from the expected time to complete the task, there is a relatively small adverse effect on production or assembly. In the case of large deviation, the adverse effect will gradually or even linearly amplify with the elapse of time. To overcome the problem, a curved time window was put forward. The curve of its penalty cost function is shown in Figure 4.

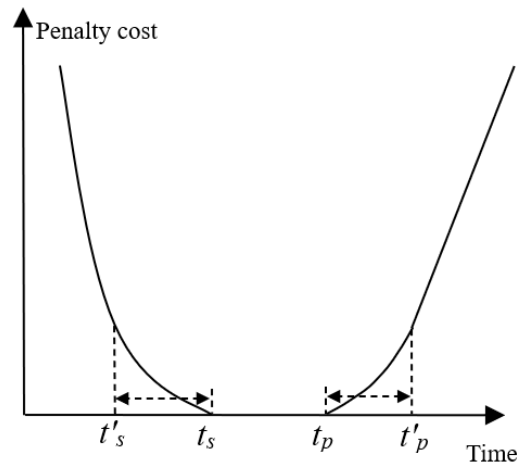

Figure 4. The curve of penalty cost function

As shown in Figure 4, the curved time window adds an acceptable service time window $\left[t_{s}{ }^{\prime}, t_{p}{ }^{\prime}\right]$ to the best service time window of the soft time window. Let $s_{i}$ be the arrival station of handling equipment $l$ at time $t_{l i}$. Then, the penalty cost it needs to pay can be expressed as:

$$
f\left(t_{l i}\right)=\left\{\begin{array}{lr}
P C_{1}\left(t_{s}^{\prime}-t_{l i}\right)+P C_{2}\left(t_{s}-t_{s}^{\prime}\right) \quad t_{l i} \leq t_{s}^{\prime} \\
P C_{2}\left(t_{s}-t_{l i}\right) & t_{s}^{\prime}<t_{l i} \leq t_{s} \\
0 & t_{s}<t_{l i} \leq t_{p} \\
P C_{3}\left(t_{l i}-t_{p}\right) & t_{p}<t_{l i} \leq t_{p}^{\prime} \\
P C_{3}\left(t_{p}^{\prime}-t_{p}\right)+P C_{4}\left(t_{l i}-t_{p}^{\prime}\right) \quad t_{p}^{\prime}<t_{l i}
\end{array}\right.
$$

where, $P C_{1}, P C_{2}, P C_{3}$, and $P C_{4}$ are the penalty costs per unit time if handling equipment $l$ arrives before $t_{s}^{\prime}$, within $\left[t_{s}^{\prime}, t_{s}\right]$, within $\left[t_{p}, t_{p}^{\prime}\right]$, and after $t_{p}^{\prime}$. The penalty cost will be zero, if handling equipment $l$ completes the handling task within $\left[t_{s}\right.$, $\left.t_{p}\right]$.

\subsection{Objective function of multi-stage path optimization}

To minimize the total time cost of logistics, the cost of material handling, and the adverse impact of handling on production and assembly in industrial job-shops, this paper establishes a multi-objective path planning and optimization model, giving full consideration to the dynamics of station demand and the maximum driving range of handling equipment.

A total of three 0-1 decision variables $v_{1-l i j}, v_{2-i l}$, and $v_{3-k l}$ were set up for the model. If $v_{1-l i j}=1$, handling equipment $l$ moves from station $s_{i}$ to station $s_{j}$; if $v_{2-i l}=1$, the task 1 of station $s_{i}$ is handled by handling equipment $l$; if $v_{3-k l}=1$, the task 2 of station $s_{k}$ is handled by handling equipment $l$.

The total time cost of logistics in an industrial job-shop consists of the planned time, the driving cost, as well as the expected time and driving cost induced by various real-time demands. Therefore, the adverse impact of path planning on production or assembly was characterized by the penalty cost function of curved time window. Then, the minimization of the total time cost can be expressed as:

$$
\begin{aligned}
& \min H=d c_{1} \sum_{i \in S} \sum_{\substack{j \in S \\
i \neq j}} \sum_{l \in L} v_{1-l i j} d_{i j}+ \\
& d c_{2} \sum_{i \in S} \sum_{\substack{j \in S \\
i \neq j}} \frac{d_{i j}}{v^{*}}+c_{k l} \sum_{k \in S} \sum_{l \in L} f^{\prime}\left(t_{l k}, D_{k l}\right)
\end{aligned}
$$

where, $S$ is the set of stations; $L$ is the set of handling equipment; $d c_{1}$ and $d c_{2}$ are the driving costs of handling equipment per unit distance and per unit time, respectively; $d_{i j}$ is the distance between stations $s_{i}$ and $s_{j} ; t_{l k}$ and $D_{l k}$ are the time and distance for handling equipment $l$ to complete task 2 of station $s_{k}$, respectively; $c_{k l}$ is the quantity of materials that task 2 of station $s_{k}$ need to be handled by handling equipment $l ; v^{*}$ is the mean velocity of handling equipment.

The time and driving cost incurred as task 2 is released by station $s_{k}$ and accepted by handling equipment $l$ can be calculated by:

$$
\begin{gathered}
f^{\prime}\left(t_{l k}, D_{k l}\right)=d c_{1} \sum_{k \in S} \sum_{l \in L} v_{3-k l} D_{k l}+ \\
d c_{2} \sum_{k \in S} \sum_{l \in L} \frac{D_{k l}}{v^{*}}
\end{gathered}
$$

The path planning must meet certain constraints. First, the number of handling equipment entering a station must equal to that leaving the station:

$$
\sum_{i \in S} v_{1-l i q}=\sum_{j \in S} v_{1-l q j}, \forall q \in S
$$

After completing the required task, a handling equipment must return to the waiting center: 


$$
\sum_{i \in S} v_{1-l 0 i}=\sum_{j \in S} v_{1-l j 0}
$$

The number of handling equipment that accepts tasks must be smaller than the total number $Q$ of available handling equipment:

$$
\sum_{i \in S} v_{1-l 0 i} \leq Q
$$

The quantity $c_{i}$ of materials in the initial task assigned by station $s_{i}$ to handling machine must fall within the rated capacity $C$ of the handling machine:

$$
\sum_{i \in S} \sum_{j \in S} \sum_{l \in L} c_{i} v_{1-l i j} \leq C, \forall i \neq j
$$

The driving distance of the handling equipment must fall within its maximum driving range $d_{\max }$ :

$$
\sum_{i \in S} \sum_{j \in S} \sum_{l \in L} v_{1-l i j} d_{i j} \leq d_{\max } \forall i \neq j
$$

The quantity of materials in dynamic tasks like task 2 must be smaller than the residual capacity of handling equipment $l$ :

$$
C-\sum_{i \in S} c_{i} v_{2-i l} \geq \sum_{k \in S} c_{k l}, \forall i \neq j
$$

During the logistics, there must be paths linking up the handling equipment and the stations it serves:

$$
\sum_{i \in S} \sum_{j \in S} \sum_{l \in L} v_{1-l i j}=v_{2-i}=v_{3-k l}, \forall i \neq j
$$

\subsection{Dynamic adjustment of handling equipment conflicts}

The handling equipment might encounter three kinds of conflicts: intersection conflict, collision, and intersection occupation. In the PLM system, the locations of intelligent handling equipment can be updated in real time, based on the data collected by RFID, WSN, and sensors. Let $\beta$ be the safe time threshold for conflict identification. Two handling equipment will have conflict at the next intersection if their real-time states satisfy:

$$
\left\{\begin{array}{c}
\left|T_{m}^{w 1}-T_{m}^{w 2}\right| \leq \beta \\
W_{m}^{w 1}=W_{m}^{w 2}
\end{array}\right.
$$

where, $T_{m}{ }^{w 1}$ and $T_{m}{ }^{w 2}$ are the arrival times of handling equipment $w 1$ and $w 2$ at intersection $m$, respectively; $W_{m}{ }^{w 1}$ and $W_{m}{ }^{w 2}$ are the identifiers of handling equipment $w 1$ and $w 2$ at intersection $m$, respectively. The conditions for collision can be expressed as:

$$
\left\{\begin{array}{l}
\left|T_{m}^{w 1}-T_{m}^{w 2}\right| \leq \beta \\
W_{m}^{w 1+1}=W_{m}^{w 2} \\
W_{m}^{w 1}=W_{m}^{w 2+1}
\end{array}\right.
$$

The conditions for intersection occupation can be expressed as:

$$
\left\{\begin{array}{l}
0 \leq\left|T_{m}^{w 1}-T_{m}^{w 2}\right| \leq T_{m}^{w 1}+\beta \\
W_{m}^{w 1}=W_{m}^{w 2}
\end{array}\right.
$$

The handling equipment in conflict states at the intersection were assigned different priorities of passing the intersection (Figure 5).
The priorities were designed based on the remaining time for the current task on each equipment. The shorter the remaining time, the higher the priority. The dynamic adjustment of priority can be expressed as:

$$
\gamma=\exp \left[\frac{R T\left(r_{i}\right)}{N(m)+1}\right]^{-1}
$$

where, $R T\left(r_{i}\right)$ is the estimated remaining time of task $r_{i} ; N(m)$ is the number of handling equipment lining up at intersection $m$.

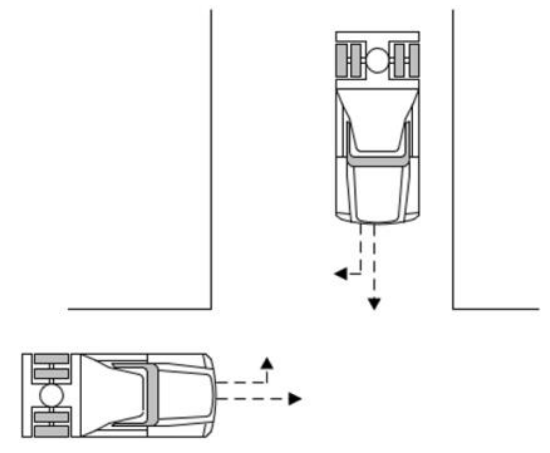

Figure 5. The intersection occupation

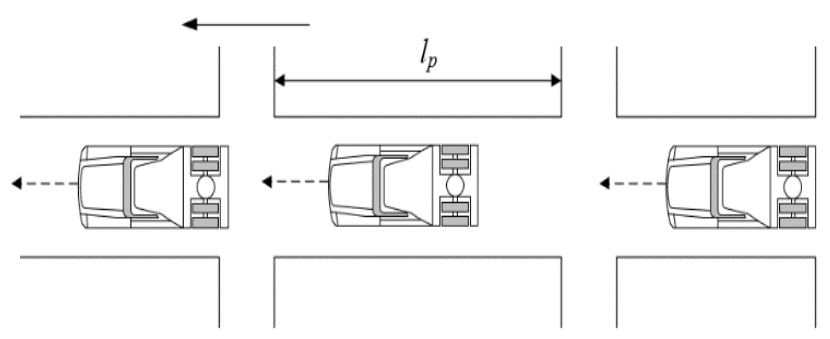

Figure 6. The congestion on the path between two intersections

Figure 6 illustrates the congestion at the path between two intersections. The congestion or non-congestion can be characterized by the number of handling equipment that can be accommodated on the path:

$$
\left\{\begin{array}{l}
N(p)=C(p)-A(p) \\
C(p)=C\left[\frac{l_{p}}{l_{H E}+\Delta d}\right]
\end{array}\right.
$$

where, $N(p)$ is the number of handling equipment that can be currently accommodated on path $p ; C(p)$ is the rated capacity of handling equipment that can be accommodated on path $p$; $A(p)$ is the number of handling equipment driving on path $p ; l_{p}$ is the length of path $p ; l_{H E}$ is the length of handling equipment; $\Delta d$ is the minimum headway between handling equipment driving on the path.

\section{LOGISTIC PATH OPTIMIZATION WNN}

To map the logistics path planning problem into a dynamic solution process of a $\mathrm{WNN}$, an $n \times n$ matrix was constructed to represent the visits to $n$ stations. Each row stands for a station, and each column stands for a node output. The serial number of a station in the station sequence visited by a handling equipment was recorded by setting the output of the node with that serial number to 1 . Table 1 provides the two-dimensional 
(2D) matrix of the effective solution to a network of six stations. The path in the table is $s_{3} \rightarrow s_{4} \rightarrow s_{5} \rightarrow s_{1} \rightarrow s_{2} \rightarrow s_{6}$.

Table 1. The 2D matrix of the effective solution

\begin{tabular}{ccccccc}
\hline Serial number & $\mathbf{1}$ & $\mathbf{2}$ & $\mathbf{3}$ & $\mathbf{4}$ & $\mathbf{5}$ & $\mathbf{6}$ \\
\hline$s_{1}$ & 0 & 0 & 0 & 1 & 0 & 0 \\
$s_{2}$ & 0 & 0 & 0 & 0 & 1 & 0 \\
$s_{3}$ & 1 & 0 & 0 & 0 & 0 & 0 \\
$s_{4}$ & 0 & 1 & 0 & 0 & 0 & 0 \\
$s_{5}$ & 0 & 0 & 1 & 0 & 0 & 0 \\
$s_{6}$ & 0 & 0 & 0 & 0 & 0 & 1 \\
\hline
\end{tabular}

Let $X_{p i}$ and $Y_{p i}$ be the input and output of node $(p, i)$, respectively. If node $i$ on path $p$ is visited, then $Y_{p i}=1$; otherwise, $Y_{p i}=0$. The energy function of the WNN can be defined as:

$$
\begin{gathered}
E=\frac{\omega_{1}}{2} \sum_{p=1}^{P} \sum_{i=1}^{P} \sum_{j=1}^{P} Y_{p i} Y_{p j}+ \\
\frac{\omega_{2}}{2} \sum_{p=1}^{P} \sum_{i=1}^{P} Y_{p i} Y_{q j}+\frac{\omega_{3}}{2}\left(\sum_{x=1}^{P} \sum_{i=1}^{P} Y_{p i}-P\right)^{2}+ \\
\frac{\omega_{4}}{2} \sum_{p=1}^{P} \sum_{q=1}^{P} \sum_{j=1}^{P} d_{p q} Y_{p i}\left(Y_{q, i+1}+Y_{q, i-1}\right)
\end{gathered}
$$

where, $\omega_{1}, \omega_{2}, \omega_{3}$, and $\omega_{4}$ are weight coefficients; $d_{p q}$ is the distance between $p$ and $q$; the first three terms are the constraints; the last term is the optimization function.

The dynamic equation of the WNN can be expressed as:

$$
\begin{gathered}
\frac{d X_{p i}}{d t}=-\frac{\partial E}{\partial Y_{p i}}=-\omega_{1}\left(\sum_{i=1}^{P} Y_{p i}-\right. \\
1)-\omega_{1}\left(\sum_{q=1}^{P} Y_{q i}-1\right)-\omega_{4} \sum_{i=1}^{P} Y_{q, i+1}
\end{gathered}
$$

The logistics path of the industrial job-shop can be solved by the WNN in the following steps:

Step 1: Initialize values and weights. Set time point to $t=0$, the value range of weight coefficients to $1.0-1.5$, and $X_{0}=0.02$.

Step 2: Calculate the distance $d_{p q}$ between path nodes, and initialize the input $X_{p i}$ to the WNN by:

$$
X_{p i}(t)=X_{0}^{\prime}+\varepsilon_{p i}
$$

where, $X_{0}^{\prime}=0.5 X_{0} \ln (P-1)$, with $P$ being the number of WNN nodes; $\varepsilon_{p i}$ is a random number within $(-1,1)$.

Step 3: Solve the dynamic equation (28), using the firstorder Euler method:

$$
X_{p i}(t+1)=X_{p i}(t)+\frac{d X_{p i}(t)}{d t} \Delta t
$$

Step 4: Calculate the output with sigmoid function:

$$
Y_{p i}(t)=\frac{1}{2}\left(1+\tanh \left(\frac{X_{p i}(t)}{X_{0}}\right)\right)
$$

Step 5: Calculate the energy function $E$ (27), evaluate the legitimacy of logistics path, and judge whether the number of iterations reaches the termination condition; If not, return to Step 3.

Step 6: Output the optimal path, the optimal energy function, the number of iterations, and the time-varying path length.

\section{SIMULATION AND RESULT ANALYSIS}

The convergence speed and accuracy of the proposed WNN were simulated on MATLAB 2016b. As shown in Figures 7 and 8 , the training error of the WNN reached the standard after 200 iterations; the actual outputs of the WNN trained on 58 sets of samples agree well with the 58 sets of data outputted from the training samples. The results demonstrate the path optimization effect of our WNN.

With fixed number of tasks and the number of handling equipment, the effects of the proposed multi-objective path planning and optimization model were compared through simulations considering and without considering conflicts at intersections. The two situations are hereinafter referred to as with and without conflicts, respectively.

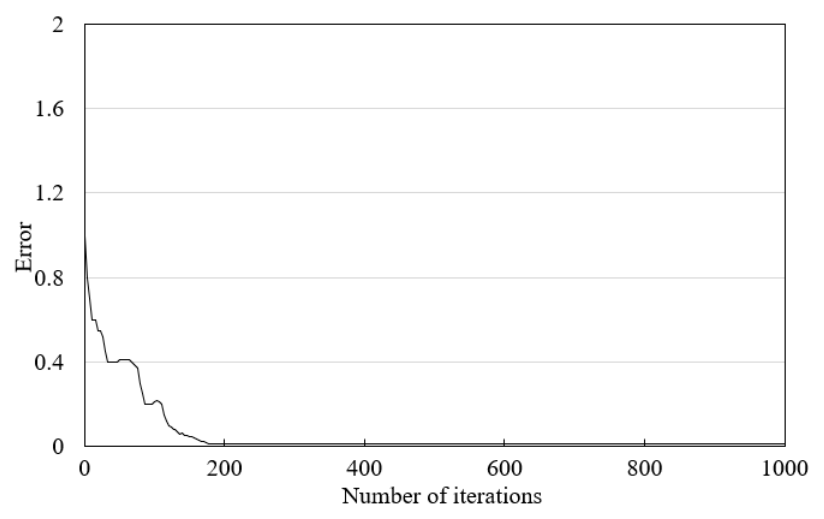

Figure 7. The training error curve of the WNN

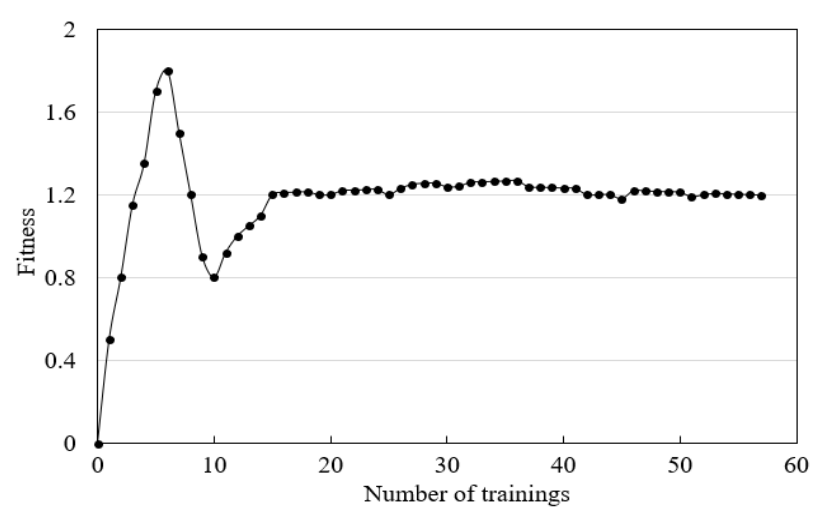

Figure 8. The fitting effect of WNN on training data

Table 2 records the simulation results with and without conflicts. It can be seen that, the waiting time of handling equipment with conflicts was always shorter than that without conflicts. As the number of tasks or handling equipment increased, the probability of conflicts between handling equipment at intersections was on the rise, and the decrements of waiting time and task completion time of handling equipment were also going up.

Figure 9 compares the time costs in 10 simulations with and without conflicts. Obviously, the consideration of conflicts reduced both waiting time and task completion time, that is, the collaborative operations of multiple equipment became more efficiency after considering the conflicts.

Table 3 compares the parameters of some handling equipment with and without conflicts at intersections. Obviously, the handling machines in the situation with conflicts (Y) had shorter idle time, higher operation frequency, smaller time cost per operation, higher effective utilization rate, and better transport capacity. These results are visually demonstrated by the curve of utilization rate of handling machines in Figure 10. 
Table 2. The simulation results with and without conflicts

\begin{tabular}{ccccc}
\hline $\begin{array}{c}\text { Simulation } \\
\text { number }\end{array}$ & $\begin{array}{c}\text { Number of } \\
\text { tasks }\end{array}$ & $\begin{array}{c}\text { Number of handling } \\
\text { equipment }\end{array}$ & $\begin{array}{c}\text { Decrement of waiting } \\
\text { time/s }\end{array}$ & $\begin{array}{c}\text { Decrement of task completion } \\
\text { time/s }\end{array}$ \\
\hline 1 & 100 & 30 & 2.0 & 5.6 \\
2 & 100 & 20 & 10.0 & 21.8 \\
3 & 150 & 25 & 5.0 & 12.9 \\
4 & 150 & 25 & 12.0 & 49.1 \\
5 & 150 & 28 & 20.0 & 56.6 \\
\hline
\end{tabular}
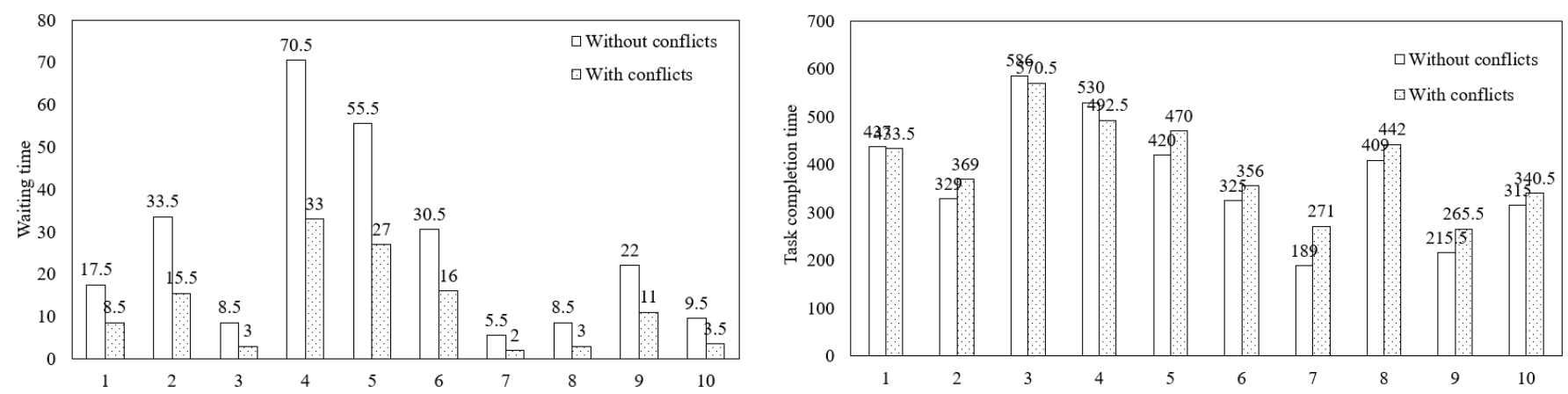

Figure 9. The time costs in 10 simulations with and without conflicts

Table 3. The parameters of some handling equipment with and without conflicts

\begin{tabular}{ccccccccccc}
\hline $\begin{array}{c}\text { Serial number of } \\
\text { handling machine }\end{array}$ & \multicolumn{2}{c}{$\begin{array}{c}\text { Idle } \\
\text { time }\end{array}$} & \multicolumn{2}{c}{$\begin{array}{c}\text { Number of } \\
\text { operations }\end{array}$} & \multicolumn{2}{c}{$\begin{array}{c}\text { Time cost per } \\
\text { operation }\end{array}$} & \multicolumn{2}{c}{$\begin{array}{c}\text { Effective utilization } \\
\text { rate }\end{array}$} & $\begin{array}{c}\text { Transport } \\
\text { capacity }\end{array}$ \\
\cline { 2 - 10 } & $\mathrm{N}$ & $\mathrm{Y}$ & $\mathrm{N}$ & $\mathrm{Y}$ & $\mathrm{N}$ & $\mathrm{Y}$ & $\mathrm{N}$ & $\mathrm{Y}$ & $\mathrm{N}$ & $\mathrm{Y}$ \\
\hline 1 & $2,163.49$ & $2,022.56$ & 425 & 461 & 57.10 & 56.56 & 88.65 & 90.56 & 70 & 80 \\
2 & $3,147.51$ & $3,122.15$ & 355 & 398 & 64.66 & 59.34 & 45.21 & 49.22 & 71 & 76 \\
3 & $3,439.38$ & $3,375.75$ & 425 & 466 & 56.95 & 51.25 & 67.17 & 71.31 & 72 & 79 \\
4 & $3,598.97$ & $3,478.21$ & 321 & 343 & 74.22 & 72.95 & 61.05 & 65.58 & 70 & 75 \\
5 & $6,427.92$ & $6,247.35$ & 215 & 287 & 75.84 & 71.41 & 48.18 & 52.19 & 81 & 86 \\
6 & 978.93 & 955.55 & 120 & 147 & 198.22 & 189.71 & 80.56 & 85.57 & 72 & 76 \\
7 & $1,095.11$ & $1,012.98$ & 153 & 187 & 157.19 & 150.53 & 80.52 & 81.98 & 74 & 85 \\
8 & $1,041.76$ & 989.27 & 198 & 209 & 124.69 & 121.68 & 83.05 & 87.43 & 75 & 83 \\
9 & $1,944.64$ & $1,792.28$ & 115 & 144 & 187.94 & 182.52 & 76.09 & 78.97 & 75 & 84 \\
10 & 901.21 & 856.91 & 127 & 136 & 192.11 & 190.17 & 71.32 & 82.65 & 72 & 84 \\
\hline
\end{tabular}

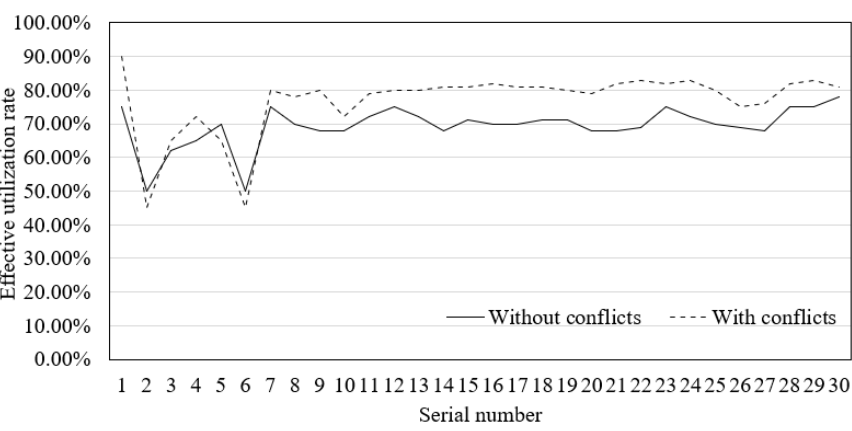

Figure 10. The utilization rates of handling machines with and without conflicts

\section{CONCLUSIONS}

Considering the excellent self-learning ability and adaptability of the AI technology of WNN, this paper proposes a WNN-based PLM model for industrial enterprises. Firstly, the architecture of the PLM system was constructed for industrial enterprises, and the scheduling and task allocation principles were designed for the collaboration of various subjects in the system. Next, a WNN was constructed for industrial PLM. Simulation results show that the WNN converged rapidly and accurately, and achieved good fitting effect on training data.

Finally, a multi-objective path planning and optimization model was constructed based on the dynamics of station demand and the maximum driving range of handling equipment. Through contrastive simulations, it is learned that, with conflicts at intersections, the proposed model can effectively improve the effective utilization rate and transport capability of handling equipment through the optimization of logistics path. The proposed model provides a novel tool for industrial enterprises to implement PLM and path planning.

\section{REFERENCES}

[1] Qu, T., Lei, S.P., Wang, Z.Z., Nie, D.X., Chen, X., Huang, G.Q. (2016). IoT-based real-time production logistics synchronization system under smart cloud manufacturing. The International Journal of Advanced Manufacturing Technology, 84(1-4): 147-164. https://doi.org/10.1007/s00170-015-7220-1

[2] Song, Y., Cao, Y.P. (2019). VMI \& TPL supply chain coordination based on evolutionary game. Journal Européen des Systèmes Automatisés, 52(2): 215-2222. https://doi.org/10.18280/jesa.520215

[3] Hou, Y., Cao, Z.J., Yang, S.L. (2019). Cloud intelligent logistics service selection based on combinatorial 
optimization algorithm. Journal Européen des Systèmes Automatisés, 52(1): 73-78 https://doi.org/10.18280/jesa.520110

[4] Bazan, E., Jaber, M.Y., Zanoni, S. (2016). A review of mathematical inventory models for reverse logistics and the future of its modeling: An environmental perspective. Applied Mathematical Modelling, 40(5-6): 4151-4178. https://doi.org/10.1016/j.apm.2015.11.027

[5] Luo, H., Wang, K., Kong, X.T., Lu, S., Qu, T. (2017). Synchronized production and logistics via ubiquitous computing technology. Robotics and ComputerIntegrated Manufacturing, 45: 99-115. https://doi.org/10.1016/j.rcim.2016.01.008

[6] Guo, Z., Zhang, Y., Zhao, X., Song, X. (2017). A timed colored petri net simulation-based self-adaptive collaboration method for production-logistics systems. $\begin{array}{lll}\text { Applied } & \text { Sciences, } & 7(3) \text { : }\end{array}$ https://doi.org/10.3390/app7030235

[7] McFarlane, D., Giannikas, V., Lu, W. (2016). Intelligent logistics: Involving the customer. Computers in Industry, 81: 105-115.

https://doi.org/10.1016/j.compind.2015.10.002

[8] Meudt, T., Metternich, J., Abele, E. (2017). Value stream mapping 4.0: Holistic examination of value stream and information logistics in production. CIRP Annals, 66(1): 413-416. https://doi.org/10.1016/j.cirp.2017.04.005

[9] Zhang, Y., Guo, Z., Lv, J., Liu, Y. (2018). A framework for smart production-logistics systems based on CPS and industrial IoT. IEEE Transactions on Industrial Informatics, 14(9): 4019-4032. https://doi.org/10.1109/TII.2018.2845683

[10] Stepanov, A.B. (2016). The application of neural network and spline wavelet models in the electroencephalogram analysis automation process. 2016 18th Conference of Open Innovations Association and Seminar on Information Security and Protection of Information Technology (FRUCT-ISPIT), St. Petersburg, pp. 321-327. https://doi.org/10.1109/FRUCTISPIT.2016.7561545

[11] Wang, F., Ma, T. (2012). Short-term traffic flow prediction based on wavelet neural network. Journal of
Ningxia Teachers University, 33(6): 60-62, 86.

[12] Masood, Z., Majeed, K., Samar, R., Raja, M.A.Z. (2017). Design of Mexican Hat Wavelet neural networks for solving Bratu type nonlinear systems. Neurocomputing, 221: 1-14. https://doi.org/10.1016/j.neucom.2016.08.079

[13] Bao, L.N., Tang, D.S., Hu, X.B., Chu, S.J. Runoff predictionmode1 based on wavelet decomposition and Arima error correction and its application. Journal of Yangtze River Scientific Research Institute, 35(12): 1821. https://doi.org/10.11988/ckyyb.20170597

[14] Akbari, Y., Nouri, K., Sadri, J., Djeddi, C., Siddiqi, I. (2017). Wavelet-based gender detection on off-line handwritten documents using probabilistic finite state automata. Image and Vision Computing, 59: 17-30. https://doi.org/10.1016/j.imavis.2016.11.017

[15] Duan, Y., Liu, F., Jiao, L., Zhao, P., Zhang, L. (2017). SAR Image segmentation based on convolutionalwavelet neural network and Markov random field. Pattern Recognition, 64: 255-267. https://doi.org/10.1016/j.patcog.2016.11.015

[16] Santhosh, M., Venkaiah, C., Kumar, D.V. (2018). Ensemble empirical mode decomposition based adaptive wavelet neural network method for wind speed prediction. Energy Conversion and Management, 168: 482-493.

https://doi.org/10.1016/j.enconman.2018.04.099

[17] Sharma, V., Yang, D., Walsh, W., Reindl, T. (2016). Short term solar irradiance forecasting using a mixed wavelet neural network. Renewable Energy, 90: 481-492. https://doi.org/10.1016/j.renene.2016.01.020

[18] Stepanov, A.B. (2017). Construction of activation functions for wavelet neural networks. 2017 XX IEEE International Conference on Soft Computing and Measurements (SCM), St. Petersburg, pp. 397-399. https://doi.org/10.1109/SCM.2017.7970597

[19] Huang, L., Wang, J. (2018). Forecasting energy fluctuation model by wavelet decomposition and stochastic recurrent wavelet neural network. Neurocomputing, 309: 70-82 https://doi.org/10.1016/j.neucom.2018.04.071 